\title{
Enhancing Expression of Nrf2-Driven Genes Protects the Blood-Brain Barrier after Brain Injury
}

\author{
Jing Zhao, Anthony N. Moore, John B. Redell, and Pramod K. Dash \\ The Vivian L. Smith Center for Neurologic Research and Departments of Neurobiology and Anatomy, and Neurosurgery, The University of Texas Medical \\ School, Houston, Texas 77225
}

The integrity of the blood- brain barrier (BBB) is critical for normal brain function, and its compromise contributes to the pathophysiology of a number of CNS diseases and injuries. Using a rodent model of brain injury, the present study examines the pathophysiology of BBB disruption. Western blot and immunohistochemical analyses indicate that brain injury causes a loss of capillary endothelial cells and tight junction proteins, two critical components of the BBB. Activation of the transcription factor NF-E2-related factor-2 (Nrf2) by sulforaphane, a naturally occurring compound present in high levels in cruciferous vegetables, significantly increased the expression of endogenous cytoprotective genes in brain tissue and microvessels as indicated by real-time PCR analysis. Postinjury administration of sulforaphane reduced the loss of endothelial cell markers and tight junction proteins and preserved BBB function. These protective effects were dependent on the activity of Nrf2. Injured rats pretreated with decoy oligonucleotides containing the binding site of Nrf2, and mice lacking the $n r f 2$ gene, did not benefit from sulforaphane administration. These findings indicate a potential therapeutic usefulness for Nrf2-activating molecules to improve the function of the neurovascular unit after injury.

Key words: brain edema; endothelial cells; neurovascular unit; tight junction proteins; TBI; capillary

\section{Introduction}

The blood-brain barrier (BBB) is critical for the maintenance of brain homeostasis and neural functions. BBB compromise contributes to the pathological changes associated with a number of neurological diseases, brain tumors, and CNS injury (Petty and Lo, 2002; DeWitt and Prough, 2003; Oby and Janigro, 2006). Loss of BBB integrity not only disrupts the function of the neurovascular unit (an assembly of brain capillaries, extracellular matrix of the basal lamina, neurons, astrocytes, oligodendrocytes, and pericytes) but also initiates secondary pathological processes, including infiltration of inflammatory cells and circulating fluid. Cerebral edema, resulting in part from the accumulation of circulating fluid, causes increased intracranial pressure leading to decreased cerebral blood flow, tissue herniation, and poor outcome (Fishman, 1975).

A layer of brain capillary endothelial cells and the contiguous tight junctions between them are major components of the BBB. Ultrastructural studies have shown that brain endothelial cells have a paucity of fenestrae and endocytotic vesicles limiting the transcellular entry of circulating molecules into the brain. In addition, the interconnection of brain capillary endothelial cells via tight junction complexes severely restricts the paracellular entry

Received April 13, 2007; revised July 10, 2007; accepted Aug. 3, 2007.

This work was supported in part National Institutes of Health Grants NS035457, NS049160, MH072933. We thank Drs. Raymond Grill and Shibani Pati for critically evaluating this manuscript. We thank Melanie Moody for technical assistance.

Correspondence should be addressed to Pramod K. Dash, Department of Neurobiology and Anatomy, The University of Texas Medical School, P.0. Box 20708, Houston, TX 77225. E-mail: p.dash@uth.tmc.edu.

DOI:10.1523/JNEUROSCI.1683-07.2007

Copyright $\odot 2007$ Society for Neuroscience 0270-6474/07/2710240-09\$15.00/0 of molecules. Although the mechanism(s) contributing to brain injury-induced $\mathrm{BBB}$ disruption has not been fully elucidated, it is likely that multiple processes, including endothelial cell death, tight junction breakdown, and decreased coupling between tight junction proteins and the cytoskeleton, could contribute to altered BBB permeability. Because multiple mechanisms may underlie $\mathrm{BBB}$ disruption after brain injury, combination treatments are likely to be more effective than single-target therapies to improve BBB function. As an alternate to combination treatments, a single agent that activates multiple cytoprotective mechanisms may also be suitable as a treatment option (Lyeth et al., 1993).

The transcription factor Nrf2 (nuclear factor E2-related factor 2) binds to the antioxidant/electrophilic response element (ARE/ EpRE) and regulates the expression of multiple cytoprotective proteins, including antioxidant and glutathione generating enzymes (Thimmulappa et al., 2002; Lee et al., 2003; Hu et al., 2004, 2006). Nrf2 is a rapidly turned-over protein that is normally sequestered in the cytoplasm via an interaction with the actinbinding protein Keap1 (Kelch-like ECH associated protein 1). Recent studies have demonstrated that Keap1 acts as a substrate adaptor protein for Cul-3 (cullin-3)-dependent ubiquitination of $\mathrm{Nrf2}$, which leads to rapid Nrf2 degradation by the proteosome system (McMahon et al., 2003; Zhang et al., 2004; Kobayashi and Yamamoto, 2006). Cellular stressors reduce the proteosomal degradation of $\mathrm{Nrf} 2$, resulting in its nuclear accumulation and increased expression of Nrf2-driven genes (Pi et al., 2003; Zhang and Hannink, 2003). In the present study, we demonstrate that systemic administration of sulforaphane, an isothiocyanate abundant in cruciferous vegetables (e.g., broccoli) but not en- 
dogenous to animals, increases the expression of Nrf2-driven genes in brain tissue and in brain microvessel preparations. Postinjury induction of Nrf2-driven genes attenuates the loss of endothelial cells and tight junction proteins and reduces BBB permeability and cerebral edema. Sequestration of Nrf2 with ARE-containing decoy oligonucleotides or loss of the $n r f 2$ gene blocks these protective effects.

\section{Materials and Methods}

Materials. Sulforaphane was purchased from LKT Laboratories (St. Paul, $\mathrm{MN})$. Anti-heme oxygenase-1 (HO-1) antibody was from Stressgen (Victoria, British Columbia, Canada). Anti-rat endothelial cell antigen-1 (RECA-1) and anti-human von Willebrand factor (vWF) antibodies were bought from AbD Serotec (Raleigh, NC), whereas anti-Nrf2 antibody was purchased from Santa Cruz Biotechnology (Santa Cruz, CA). Anti-claudin-5 antibody, anti-occludin antibody, SuperScript II Reverse Transcriptase, and NanoOrange were bought from Invitrogen (Carlsbad, CA). AmpliTaq DNA Polymerase was obtained from Applied Biosystems (Foster City, CA).

Subjects. Male Sprague Dawley rats (Harlan, Indianapolis, IN) weighing 260-300 g, C57BL/6 mice (Harlan), and $n r f 2^{-/-}$mice (generously provided by Dr. Y. W. Kan, University of California, San Francisco, San Francisco, CA) were housed under temperature-controlled conditions with a $12 \mathrm{~h}$ light/dark cycle and ad libitum access to water and food. Animal protocols were approved by the Institutional Animal Welfare Committee and were in compliance with the National Institutes of Health Guide for Care and Use of Laboratory Animals.

Brain injury. A controlled cortical impact model was used to cause brain injury as described previously (Dixon et al., 1991; Meaney et al., 1994; Dash et al., 1995). Rats were initially anesthetized using 5\% isoflurane with a $1: 1 \mathrm{~N}_{2} \mathrm{O} / \mathrm{O}_{2}$ mixture and then maintained with a $2.5 \%$ isoflurane with 1:1 $\mathrm{N}_{2} \mathrm{O} / \mathrm{O}_{2}$ mixture via a face mask. Animals were mounted on the stereotaxic frame secured by ear bars and an incisor bar. The head was held in a horizontal plane with respect to the interaural line. A midline incision was made, and a 6-mm-diameter craniectomy was made midway between the bregma and the lambda on the right side, with the medial edge of the craniectomy $1 \mathrm{~mm}$ lateral to midline. The injury was caused by a pneumatic piston at an angle of $10^{\circ}$ from the vertical plane at $6 \mathrm{~m} / \mathrm{s}$ velocity with $1.8 \mathrm{~mm}$ deformation. After injury, the incision was closed with wound clips. Sham-operated animals received all the aforementioned surgical procedures except the craniectomy and impact injury. Core body temperature was maintained at $36.0-36.5^{\circ} \mathrm{C}$ during the surgery using a rectal thermometer coupled to a heating pad. Mice were injured at $4 \mathrm{~m} / \mathrm{s}$ velocity with $1.7 \mathrm{~mm}$ deformation. After surgery, all animals were allowed to completely recover from the anesthesia in a warm chamber before being transferred to their home cages.

Electrophoretic mobility shift assays. Double-stranded oligonucleotide probes were $\left[{ }^{32} \mathrm{P}\right]$-labeled using T4 polynucleotide kinase, and cortical nuclear extracts for electrophoretic mobility shift assays (EMSAs) were prepared as described previously (Dash et al., 1995). Nuclear extracts (4 or $10 \mu \mathrm{g}$ ) were combined with $0.5 \mathrm{ng}$ of labeled probes in a buffer containing $10 \mathrm{~mm}$ Tris, pH 7.9, $5 \mathrm{~mm} \mathrm{MgCl}_{2}, 0.5 \mathrm{~mm}$ EDTA, $100 \mathrm{~mm} \mathrm{NaCl}, 50$ $\mathrm{mm} \mathrm{KCl}, 5 \%$ sucrose, $5 \%$ glycerol, $0.5 \mathrm{~mm}$ dithiothreitol (DTT), 0.5 $\mu \mathrm{g}$ of sonicated salmon sperm DNA, and $0.5 \mu \mathrm{g}$ of poly(dI-dC). Cold competitors were added simultaneously, and binding was allowed to occur for $20 \mathrm{~min}$ at room temperature. The oligonucleotide probe bound to nuclear proteins were separated from free probe in a $6 \%$ polyacrylamide, $0.5 \times$ Tris-borate EDTA gel and visualized by autoradiography.

Shift-Western blot analysis. After the separation of the free and bound probes by EMSA, gels were transferred to a nitrocellulose membrane in a buffer containing $48 \mathrm{~mm}$ Tris, pH 8.5, $39 \mathrm{~mm}$ glycine, and 20\% methanol using a semidry transfer apparatus (Millipore, Billerica, MA). After overnight blocking in Tris-buffered saline supplemented with $0.1 \%$ Tween-20 (TBST) and 5\% bovine serum albumin, Western blot analysis was performed using $0.5 \mu \mathrm{g} / \mathrm{ml} \mathrm{Nrf2}$ antibodies. Immunoreactivity was visualized using a CDP-Star chemiluminescent substrate (New England
Biolabs, Beverly, MA). A Hybond- ${ }^{+}$membrane (GE Healthcare, Piscataway, NJ) placed under the nitrocellulose captured the DNA probe and was used for autoradiography. Migration of the protein and DNA signals were compared by overlaying the resultant films.

Intracerebroventricular infusion. The following oligonucleotides were synthesized by Integrated DNA Technologies (Coralville, IA) and used in these studies: ARE, 5'-CTAATGGTGACAAAGCAACTTT-3' and its compliment; AREmut, 5' -CGACTGCCTTCAAAATAACTTT-3' and its compliment. Underlining indicates the ARE core binding sequence. Double-stranded decoy oligonucleotides $(20 \mu \mathrm{g} / \mu \mathrm{l})$ were prepared by annealing complimentary single strands in sterile saline. Rats were anesthetized as described above, and $10 \mu \mathrm{l}$ of oligonucleotides were infused to the right ventricle $(1.8 \mathrm{~mm}$ lateral, $0.8 \mathrm{~mm}$ posterior to bregma, $3.6 \mathrm{~mm}$ depth from the skull surface) using a 30 gauge needle at a rate of $1 \mu \mathrm{l} / \mathrm{min}$. Oligonucleotides were infused at $24 \mathrm{~h}$ and again at $2 \mathrm{~h}$ before intraperitoneal sulforaphane administration.

Microvessel preparation. Brain microvessels were purified essentially as described by Wagnerova et al. (2002). Animals were given vehicle or sulforaphane (intraperitoneally) and killed by decapitation $18 \mathrm{~h}$ later, and the brains were removed quickly. The brains were weighed, minced in 20 vol of high-glucose DMEM, and homogenized with 20 strokes of a glass-Teflon homogenizer shaved to a nominal clearance of $0.20 \mathrm{~mm}$. The homogenate was passed through $125 \mu \mathrm{m}$ nylon mesh, the cleared filtrate was centrifuged at $800 \times g$ for $5 \mathrm{~min}$, and the pellet was gently resuspended in $15 \mathrm{ml}$ of $15 \%$ dextran-70 in DMEM. The suspension was centrifuged at $4500 \times g$ for $15 \mathrm{~min}$, and the supernatant was completely aspirated. The pellet was resuspended in $3 \mathrm{ml}$ of PBS and filtered through $40 \mu \mathrm{m}$ nylon mesh to capture microvessels. The isolated microvessels were stored at $-80^{\circ} \mathrm{C}$ for later use in real-time PCR.

Real-time PCR. Naive animals were given sulforaphane or vehicle (intraperitoneal) and killed $18 \mathrm{~h}$ later. For injury studies, animals received sulforaphane or vehicle $6 \mathrm{~h}$ after injury and were killed $24 \mathrm{~h}$ after injury. Ipsilateral peri-core parietal cortex (injured), or a corresponding region of the parietal cortex (uninjured), was quickly dissected out and frozen in dry ice. The tissue was homogenized in $1.5 \mathrm{ml}$ of TriZol (Invitrogen) per $100 \mathrm{mg}$ tissue, followed by addition of chloroform (1:5) and incubation on ice for $20 \mathrm{~min}$. The homogenate was centrifuged at $14,000 \times g$ for 30 $\mathrm{min}$, and total RNA from the aqueous layer was precipitated with isopropanol. One microgram total RNA was reverse transcribed for $2 \mathrm{~h}$ at $37^{\circ} \mathrm{C}$ in a $20 \mu \mathrm{l}$ mixture containing $50 \mathrm{~mm}$ Tris- $\mathrm{HCl}, \mathrm{pH} 8.3,75 \mathrm{~mm} \mathrm{KCl}, 3.0$ $\mathrm{mm} \mathrm{MgCl}_{2}, 10 \mathrm{~mm}$ DTT, $2.5 \mu \mathrm{M}$ random hexamer, 1.0 mM each dNTP, 20 U of Rnasin, and $200 \mathrm{U}$ of Superscript II reverse transcriptase. The level of expression of each target gene was quantified using a Bio-Rad (Hercules, CA) iCycler real-time PCR system. Reactions were performed in triplicate. Each $30 \mu \mathrm{l}$ reaction mixture consisted of $3.0 \mu \mathrm{l}$ of the cDNA and 18 mм Tris- $\mathrm{HCl}, \mathrm{pH} 8.3,55 \mathrm{~mm} \mathrm{KCl}, 2.0 \mathrm{~mm} \mathrm{MgCl}_{2}, 0.2 \mathrm{~mm}$ of each dNTP, $0.5 \mu \mathrm{M}$ of each primer, $10 \mathrm{~nm}$ fluorescein, 1:75,000 dilution of Sybr green $\mathrm{I}$, and $2 \mathrm{U}$ of AmpliTaq DNA polymerase. The following primer pairs were used for target mRNA amplification: glutathione $S$-transferase $\alpha 3$ (GST $\alpha 3$ ), rat: forward, 5' -AGTCCTTCACTACTTCGATGGCAG-3'; reverse, 5'-CACTTGCTGGAACATCAAACTCC-3'; HO-1, rat: forward, 5'-AAGGAGGTGCACATCCGTGCA-3'; reverse, 5'-ATGTTGAGCAGGAAGGCGGTC- $3^{\prime} ; \beta$-actin, rat: forward, $5^{\prime}$-CCCCATTGAACACGGCATT-3'; reverse, 5' -CATCTTTTCACGGTTGGCCTTA-3'; glutathione peroxidase (GPx), mouse: forward, 5' - GACTGGTGGTGCTCGGTT- 3'; reverse, 5' -ACTTGAGGGAATTCAGAATCTCTTC3'; $\beta$-actin, mouse: forward, 5'- GCTTCTTTGCAGCTCCTTCGT-3'; reverse, 5'- ATATCGTCATCCATGGCGAAC-3'. The amplification protocol consisted of $1 \mathrm{cycle}$ at $95^{\circ} \mathrm{C}$ for $3 \mathrm{~min}$ followed by 40 cycles at $95^{\circ} \mathrm{C}$ for $30 \mathrm{~s}, 58^{\circ} \mathrm{C}$ for $30 \mathrm{~s}$, and then $72^{\circ} \mathrm{C}$ for $30 \mathrm{~s}$. The melt-curve protocol, performed at the end of the amplification, consisted of 80 cycles beginning at $55^{\circ} \mathrm{C}$ for $10 \mathrm{~s}$, after which the temperature was increased by $0.5^{\circ} \mathrm{C} /$ cycle. Data was analyzed using the iCycler iQ Real-Time Detection System software (Redell et al., 2003). A standard curve for each target gene was generated to determine the linear range and amplification efficiency of each sample. The threshold cycle of each experimental sample was fitted to the standard curve to calculate the relative abundance of each target in the cDNA preparation. mRNA 
abundance was normalized to $\beta$-actin levels and presented in terms of input total RNA.

Western blots. Rats were killed at the indicated times, and the brains were quickly removed and submerged under ice-cold artificial CSF. The parietal cortex tissue from the peri-core region was dissected and homogenized in a buffer consisting of $10 \mathrm{~mm}$ Tris, pH 7.4, 1 mM EGTA, $1 \mathrm{~mm}$ EDTA, $0.1 \mu \mathrm{M}$ okadaic acid, $1 \mathrm{~mm}$ PMSF, and $10 \mu \mathrm{g} / \mathrm{ml}$ leupeptin. The protein concentration of each sample was measured by NanoOrange assay. After being boiled in Laemli's buffer for $3 \mathrm{~min}$, equal amount of protein samples were loaded and resolved on a $10 \%$ Tris/tricine SDSPAGE gel and then transferred to a polyvinylidene difluoride membrane. The membrane was blocked in 5\% BSA in TBST overnight and then incubated with primary antibody diluted in 2\% BSA in TBST for $3 \mathrm{~h}$ at room temperature. Immunoreactivity was assessed using an alkaline phosphatase-conjugated secondary antibody and the CPD-Star chemiluminescence substrate (New England Biolabs).

Immunohistochemistry and fluorescence intensity quantification. Animals were transcardially perfused with $1 \times$ PBS, followed by $4 \%$ paraformaldehyde in PBS $24 \mathrm{~h}$ after traumatic brain injury (TBI). Brains were removed, postfixed in $4 \%$ paraformaldehyde/PBS at $4^{\circ} \mathrm{C}$ overnight, and incubated in $30 \%$ sucrose for cryoprotection. Coronal sections $(40 \mu \mathrm{m})$ were prepared on a cryostat. The sections were blocked in $5 \%$ goat serum in TBS with $0.25 \%$ Triton X-100 (TBS-Tx) at room temperature for $1 \mathrm{~h}$, followed by incubation with primary antibodies in $2.5 \%$ goat serum in TBS-Tx at $4^{\circ} \mathrm{C}$ for $48 \mathrm{~h}$ and then with species-specific secondary antibodies in $2.5 \%$ goat serum in TBS-Tx for $3 \mathrm{~h}$ at room temperature. For immunostaining with occludin, claudin-5, and zona occluden-1 (ZO-1) antibodies, fresh-frozen and methanol fixed tissue were used. Briefly, after decapitation, brains were removed and quickly frozen in $-80^{\circ} \mathrm{C}$ isopentane. Ten-micrometer-thick coronal sections were prepared and mounted directly on slides. After air drying, the sections were fixed with $100 \%$ methanol for $20 \mathrm{~min}$ at $-20^{\circ} \mathrm{C}$ and then rinsed in TBS-Tx for 20 min. Immunostaining was performed as described above. Fluorescence intensity was quantified essentially as described previously (Masliah et al., 1992; Alford et al., 1994; Verschure et al., 1994). Briefly, images of immunofluorescence were captured using a Bio-Rad MRC 1024 confocal microscope and Olympus Optical (Center Valley, PA) BX 50WI camera. The parameters used for image acquisition (including laser power, iris size, brightness, offset, etc.) were preset to minimize the background and optimize the signal using a tissue section from an injured animal. These parameters were kept constant across all subsequent groups. A stack of pictures was generated for each section by scanning through the section at a step thickness of $0.80 \mu \mathrm{m}$ along the $z$-axis. MetaMorph 6.1 software (Universal Imaging, Downingtown, PA) was used to determine the fluorescence intensity based on the stack of pictures. The sections containing the contusion core corresponded to $2.0 \mathrm{~mm}$ caudal to bregma level, whereas the sections representing the penumbra were taken from $1.0 \mathrm{~mm}$ caudal to bregma level. Three nonoverlapping regions $(844 \times 633 \mu \mathrm{m})$ in the ipsilateral cortex from each section and two sections from each animal were used for imaging. The fluorescence intensities of the three regions were averaged for each section, and the two sections were averaged for each animal.

NADPH:quinone oxidoreductase 1 activity assay. Animals were killed $18 \mathrm{~h}$ after administration of sulforaphane or vehicle, and parietal cortical tissues were quickly dissected out, frozen on dry ice, and stored at $-80^{\circ} \mathrm{C}$ until used. The tissue was homogenized in ice-cold buffer containing 25 mм Tris- $\mathrm{HCl}, \mathrm{pH} 7.4$, and $125 \mathrm{~mm}$ sucrose, sonicated for $5 \mathrm{~s}$, and then left on ice for $10 \mathrm{~min}$. The homogenate was centrifuged at $20,000 \times g$ for 20 $\min$ at $4^{\circ} \mathrm{C}$, and the supernatant was used for enzyme activity measurements. NADPH:quinone oxidoreductase 1 (NQO1) activity was determined by monitoring the reduction of the substrate 2,6dichloroindophenol (DCIP). The protein sample was added to a solution containing $25 \mathrm{~mm}$ Tris- $\mathrm{HCl}, \mathrm{pH} 7.4,0.006 \%$ bovine serum albumin, $0.01 \%$ Tween 20, $5 \mu \mathrm{M}$ flavin adenine dinucleotide, $0.2 \mathrm{~mm}$ nicotinamide adenine dinucleotide, and $40 \mu \mathrm{M}$ DCIP. The change in absorbance at 600 $\mathrm{nm}$ was monitored in a spectrophotometer every $10 \mathrm{~s}$ over $5 \mathrm{~min}$ at room temperature. Reaction conditions (protein and substrate concentrations) that yielded a linear change in absorbance for up to 5 min were initially established. Specific activity was calculated using the rate of change in absorbance over the timeframe of 1-3 min.

Measurement of blood-brain barrier permeability. BBB permeability was assessed at the indicated times by measuring the extravasation of either Evans Blue (EB) dye or fluorescein as described previously (Shapira et al., 1993; Shohami et al., 1994). Three percent EB or $2 \%$ sodium fluorescein in saline was injected slowly through the jugular vein $(4 \mathrm{ml} /$ $\mathrm{kg}$ ) and allowed to circulate for $1.5 \mathrm{~h}$. For EB measurements, animals were transcardially perfused with PBS, followed by PBS containing $4 \%$ paraformaldehyde. Brains were removed, and ipsilateral hemispheres were cut into 2 -mm-thick sections and incubated in $5 \mathrm{ml}$ of formamide at $55^{\circ} \mathrm{C}$ for $24 \mathrm{~h}$ (mouse brain slices were incubated in $1 \mathrm{ml}$ of formamide). After incubation, the formamide solution was cleared by centrifugation at $20,000 \times g$ for $20 \mathrm{~min}$. The supernatant was collected, and the optical density at $620 \mathrm{~nm}$ was measured to determine the relative amount of EB dye in each sample. For fluorescein measurements, brains were removed immediately after being perfused with PBS. Ipsilateral hemisphere was homogenized in 50\% trichloroacetic acid and centrifuged at 20,000 $\times g$ for $20 \mathrm{~min}$. The supernatant solution was collected and neutralized with $\mathrm{NaOH}$, and the fluorescence was measured using a fluorometer with $\lambda_{\mathrm{EX}}$ of $485 \mathrm{~nm}$ and $\lambda_{\mathrm{EM}}$ of $530 \mathrm{~nm}$.

Brain edema measurement. Percentage of brain water content was determined using the wet-dry method as described previously (McIntosh et al., 1990; Shohami et al., 1993). Animals were injured, and sulforaphane or vehicle was administered (intraperitoneally) 15 min later. Animals were killed by decapitation $24 \mathrm{~h}$ after injury, the brains were quickly removed, and the cerebellums were discarded. Ipsilateral and contralateral hemispheres were separated, and the wet weight of each hemisphere was measured with an analytical balance. The tissues were then completely dried in a desiccating oven at $100^{\circ} \mathrm{C}$ for $48 \mathrm{~h}$, and the dry weight of each hemisphere was recorded. The percentage of water content $\left(\% \mathrm{H}_{2} \mathrm{O}\right)$ is calculated for each hemisphere as follows: $\% \mathrm{H}_{2} \mathrm{O}=[$ (wet weight - dry weight)/wet weight] $\times 100$.

Statistical analysis. SigmaStat (Systat Software, San Jose, CA) was used to perform the statistical analysis of all the results. A one-way ANOVA followed by appropriate post hoc analysis was used to compare single variable data. A two-way ANOVA was used to compare between groups for time course studies. Data are presented as the mean \pm SEM. Differences were considered statistically significant at $p<0.05$.

\section{Results}

\section{Nrf2-driven genes in the brain and brain microvessels are induced by systemic sulforaphane administration}

Recent microarray studies have shown that systemic administration of sulforaphane increases the mRNA levels of several detoxifying and cytoprotective genes in mouse liver ( $\mathrm{Hu}$ et al., 2004, 2006). Consistent with these reports, we found that systemic administration of sulforaphane $(5 \mathrm{mg} / \mathrm{kg}$, i.p.) to uninjured rats significantly increased the mRNA levels for GST $\alpha 3$, an Nrf2driven gene, in the liver compared with vehicle controls $18 \mathrm{~h}$ after treatment as indicated by real-time PCR (vehicle, 950.0 \pm 106.6 ng; sulforaphane, $1490.0 \pm 185.3 \mathrm{ng} ; n=4$ per condition; $p<$ 0.05; data not shown). To examine whether sulforaphane can increase the expression of Nrf2-driven genes in brain tissue and microvessels, we performed real-time PCR analysis for selected target genes. RNA was prepared from parietal cortex tissue punches or isolated microvessels $18 \mathrm{~h}$ after sulforaphane $(5 \mathrm{mg} /$ $\mathrm{kg}$, i.p.) or vehicle administration. Figure $1 \mathrm{~A}$ (left) shows that sulforaphane increases the expression of GST $\alpha 3$ in the parietal cortex from uninjured animals ( $n=3$ per condition). To examine whether sulforaphane is also effective in the injured brain, drug or vehicle was administered $6 \mathrm{~h}$ after injury $(n=4$ per condition), and cortical tissue surrounding the contusion was harvested at $24 \mathrm{~h}$ for mRNA analysis. A $6 \mathrm{~h}$ postinjury time point was chosen for sulforaphane administration based on previous clinical studies indicating that pharmacological treatments could 
A

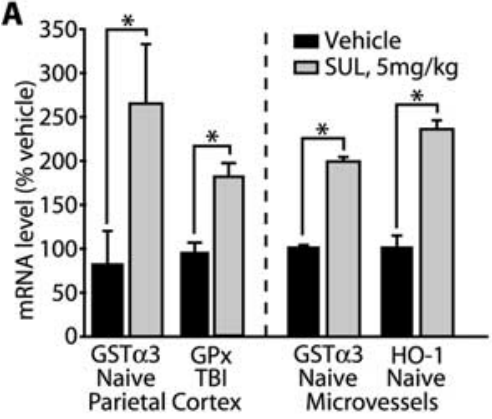

D

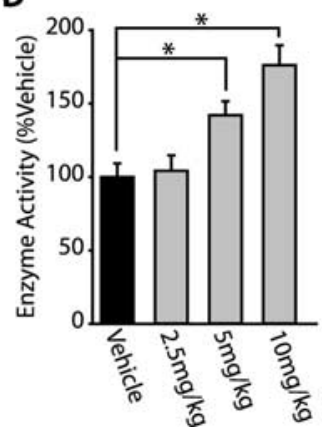

B

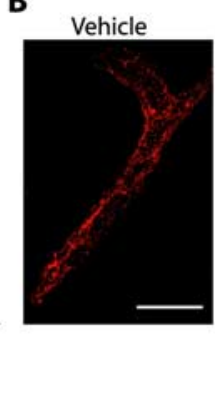

E

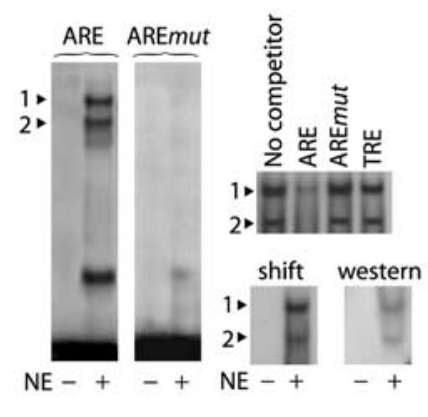
SUL, $5 \mathrm{mg} / \mathrm{kg}$

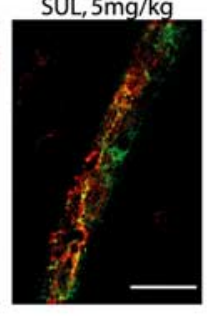

$\mathbf{F}$
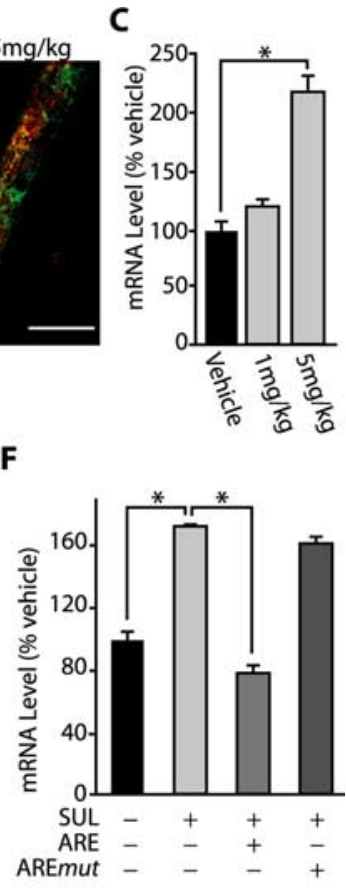

Figure 1. Nrf2-driven genes in the brain and brain microvessels are induced by systemic sulforaphane administration. $\boldsymbol{A}$, Left, Sulforaphane increases the mRNA level of Nrf2-driven genes in the parietal cortex from both uninjured and injured animals. Sulforaphane $(5 \mathrm{mg} / \mathrm{kg}$ ) was administered $6 \mathrm{~h}$ after injury and tissue harvested for mRNA analysis at $24 \mathrm{~h}$. $\boldsymbol{A}$, Right, Sulforaphane (SUL) increases mRNA levels of Nrf2-driven genes in purified brain microvessels from uninjured animals. $\boldsymbol{B}$, Representative confocal images showing enhanced H0-1 immunoreactivity (green) in brain microvessels (indicated by vWF immunoreactivity; red) $18 \mathrm{~h}$ after $5 \mathrm{mg} / \mathrm{kg}$ sulforaphane treatment. C, Sulforaphane at $5 \mathrm{mg} / \mathrm{kg}$, but not $1 \mathrm{mg} / \mathrm{kg}$, increases GST $\alpha 3 \mathrm{mRNA}$ level in cortical tissue. $\boldsymbol{D}$, A dose-dependent increase in NADPH:quinone oxidoreductase 1 enzymatic activity is observed $18 \mathrm{~h}$ after sulforaphane treatment. $\boldsymbol{E}$, EMSA and shift-Western assays showing the specificity and binding of Nrf2 to the decoy (ARE), but not the mutant (AREmut), oligonucleotide. NE, Nuclear extract. $\boldsymbol{F}$, Summary data showing that intracerebroventricular infusion of the decoy oligonucleotide (ARE), but not the mutated oligonucleotide (AREmut), blocked the induction of GST $\alpha 3 \mathrm{mRNA} 18 \mathrm{~h}$ after administration of $5 \mathrm{mg} / \mathrm{kg}$ sulforaphane in uninjured animals. Data are presented as the mean \pm SEM. * $p<0.05$. Scale bars, 50 $\mu \mathrm{m}$.

not be easily initiated before this time point. Real-time PCR measurements indicated a significant increase of GPx mRNA in sulforaphane-treated animals (Fig. $1 A$, left).

When purified brain microvessel preparations from sulforaphane- and vehicle-treated uninjured animals were assessed for Nrf2-driven gene expression, the mRNA levels of GST $\alpha 3$ and HO- 1 were found to be increased by $5 \mathrm{mg} / \mathrm{kg}$ sulforaphane (Fig. $1 A$, right; $n=4$ per condition). Consistent with the observed increase in HO-1 mRNA, Figure $1 B$ shows that the immunoreactivity for HO-1 (green) is enhanced in microvessels identified by colabeling with the endothelial cell marker vWF (red). Although $5 \mathrm{mg} / \mathrm{kg}$ sulforaphane significantly increased GST $\alpha 3$ mRNA in the brain $18 \mathrm{~h}$ after administration, a dose of 1 $\mathrm{mg} / \mathrm{kg}$ did not (Fig. $1 C ; n=4$ per condition). The levels of $\beta$-actin, used as an internal control, did not reveal any significant differences in overall mRNA level between the samples in any of the above experiments (data not shown). In addition to increasing HO-1 mRNA and protein (Fig. $1 A, B$ ), sulforaphane treatment also induced the enzyme activity of NQO1, a representative Nrf2-driven gene (Thimmulappa et al., 2002), in a dosedependent manner (Fig. 1D).

To confirm that the gene induction we observed is dependent on Nrf2, double-stranded oligonucleotides containing either the binding site for Nrf2 (ARE) or a mutated Nrf2 binding site (AREmut), were synthesized and used as decoys. We and others have previously demonstrated that infusion of double-stranded oligo- nucleotides into the brain can be used to sequester a specific transcription factor, or transcription factor complex, and perturb target gene regulation (Qiu et al., 2004; Dash et al., 2005). Before using these oligonucleotides in vivo, we characterized their binding in vitro using EMSAs. Figure $1 E$ shows representative EMSAs using the ARE and AREmut oligonucleotides as probes. The left panel shows that incubation of nuclear extract from rat parietal cortex tissue with the radiolabeled ARE oligonucleotide gave rise to two low mobility bands (arrowheads). In contrast, no binding was observed with the AREmut probe. Assays using ARE oligonucleotides as the probe and unlabeled oligonucleotide competitors demonstrated that the binding of these two bands was specific to the ARE sequence. This binding could not be competed with either excess mutant oligonucleotide or an oligonucleotide containing the nonrelated binding site for activator protein-1 complexes [TRE (12-Otetradecanoylphorbol-13-acetate response element)]. To further examine the presence of Nrf2 protein in the retarded bands, EMSA followed by Western blotting (shift-Western) using an antibody against Nrf2 was performed. Results shown in Figure $1 E$, right bottom, indicate that the two retarded bands were immunopositive for Nrf2, consistent with their binding to the ARE probe. Satisfied by the specificity of the oligonucleotides, we next examined whether their infusion would block the ability of sulforaphane to increase Nrf2driven gene expression in the brain. Guided by the concentration of decoy oligonucleotides used in previous reports, uninjured rats were infused with $200 \mu \mathrm{g}$ of either the ARE or AREmut oligonucleotides into the lateral ventricle 24 and $2 \mathrm{~h}$ before sulforaphane intraperitoneal injection. Figure $1 F$ shows that the increase in GST $\alpha 3$ mRNA levels measured $18 \mathrm{~h}$ after sulforaphane injection was blocked by intracerebroventricular infusion of the ARE, but not the AREmut, oligonucleotides $(n=4$ per condition), indicating that Nrf2 is required for its induction.

\section{Sulforaphane attenuates the loss of tight junction proteins and endothelial cells after brain injury}

Using total protein homogenates prepared from cortical tissue surrounding the contusion core at increasing time points after injury, levels of the tight junction proteins occludin and claudin- 5 were determined by Western blotting. Figure $2 \mathrm{~A}$ shows a picture of a representative Western blot and summary data demonstrating that injury causes a significant decrease in occludin immunoreactivity. Occludin immunoreactivity was decreased for up to $14 \mathrm{~d}$ after injury, with a maximal effect observed at the $24 \mathrm{~h}$ and $3 \mathrm{~d}$ after injury. Similarly, the levels of claudin-5 were significantly reduced at $24 \mathrm{~h}$ and $3 \mathrm{~d}$ after injury (Fig. $2 \mathrm{~B}$; $n=3$ per time point). We sought to determine whether postinjury sulforaphane administration could abate the loss of these tight junction proteins. Animals were intraperitoneally injected with either $5 \mathrm{mg} / \mathrm{kg}$ sulforaphane or vehicle $6 \mathrm{~h}$ after injury, and 
A

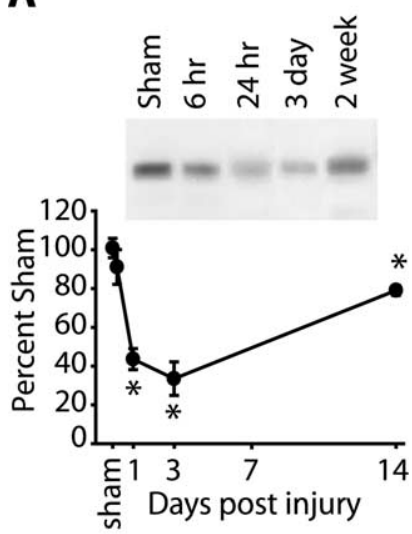

B

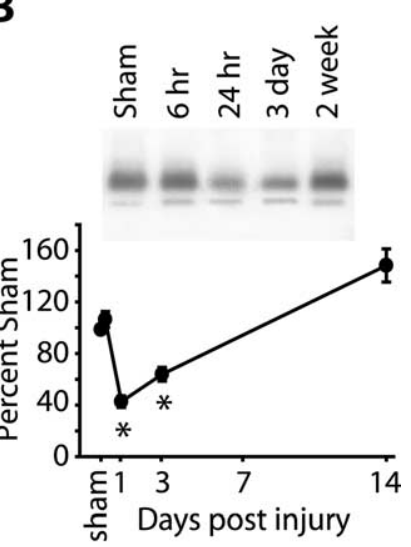

C

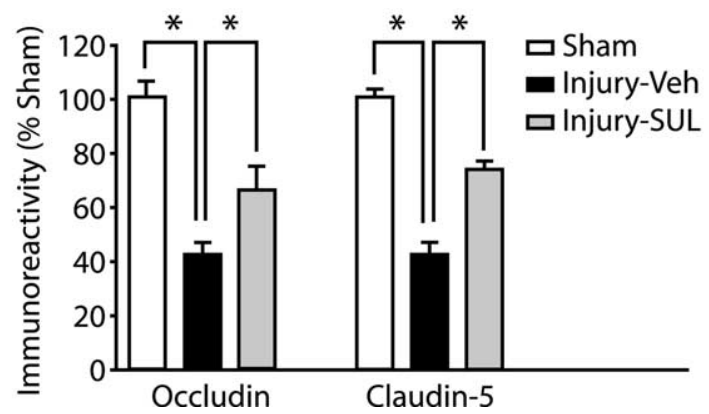

Figure 2. Traumatic brain injury causes loss of tight junction proteins. Representative Western blots and summary data for occludin $(\boldsymbol{A})$ and claudin-5 $(\boldsymbol{B})$ immunoreactivities after injury. Occludin was significantly decreased from 1 to $14 \mathrm{~d}$ after injury, whereas claudin-5 was significantly decrease at the 1 and $3 \mathrm{~d}$ time points. C, Quantification of Western blots indicating that $6 \mathrm{~h}$ postinjury administration of $5 \mathrm{mg} / \mathrm{kg}$ sulforaphane (SUL) partially preserved occludin and claudin- 5 immunoreactivity at $24 \mathrm{~h}$. Veh, Vehicle. Data are presented as the mean \pm SEM. ${ }^{*} p<$ 0.05 .

total protein extracts from the parietal cortex surrounding the injury core were prepared $24 \mathrm{~h}$ after injury. Western blot analysis revealed that the loss of occludin and claudin-5 immunoreactivities as a result of injury was significantly attenuated in rats treated with sulforaphane (Fig. $2 C ; n=5$ per condition).

Figure $3 A$ shows representative confocal images of occludin immunoreactivity from three groups (Sham, Injury-Veh, and Injury-SUL). Abundant vessel-like structures can be seen in the tissue section from the sham control (Sham, top row), the number of which dramatically decreases as a result of injury (InjuryVeh, top row). Compared with the vehicle-treated injured animal, the representative image from an injured animal treated with sulforaphane has more occludin-immunopositive vessellike structures (Injury-SUL, top row). High-magnification confocal images from tissue sections double labeled with occludin (green) and vWF (red) demonstrate that the normally contiguous expression pattern of occludin (along the vessel tube marking the endothelial cell borders) on intact vessels is disrupted as a result of injury (bottom row, Sham vs Injury-Veh). The contiguous staining pattern is partially restored in animals treated with sulforaphane (Injury-SUL, bottom row). Quantification of occludin fluorescence intensity confirmed our Western blot findings that sulforaphane treatment protects against the postinjury loss of occludin. Similarly, claudin-5 and ZO-1 fluorescence intensities in sulforaphane-treated injured animals are also increased relative to vehicle-treated injured animals (Fig. $3 B ; n=4$ per condition). To examine whether the sulforaphane-mediated protection of tight junction protein is through induction of their mRNA expression, we measured occludin and claudin- 5 mRNA levels by real-time PCR. No significant changes in occludin or claudin-5 mRNAs were observed in response to sulforaphane treatment (data not shown).

To examine endothelial cell loss after injury, the levels of the endothelial cell markers RECA-1 and vWF were examined by immunohistochemistry. Representative confocal images for RECA-1 immunoreactivity from a sham, an injured animal receiving vehicle, and an injured animal receiving sulforaphane are shown in Figure 3C (low magnification, top row; high magnification, middle row). The summary results for integrated fluorescence intensity indicate that brain injury markedly reduces RECA- 1 and vWF immunoreactivities and that postinjury sulforaphane administration can partially attenuate these losses (Fig. 3D;n=7 per condition). The expression of endothelial barrier antigen (EBA), an immunohistochemical marker whose expression correlates with BBB integrity (Rosenstein et al., 1992), was also examined to assess vascular integrity. Figure $3 C$ (bottom row) shows representative confocal images demonstrating that, compared with tissue sections from a sham-operated animal, brain injury markedly decreases EBA immunoreactivity. Postinjury administration of $5 \mathrm{mg} / \mathrm{kg}$ sulforaphane partially attenuated the loss of EBA immunoreactivity (Fig. 3D).

\section{Activation of Nrf2 decreases blood-brain barrier permeability after injury}

To directly examine changes in BBB permeability after injury, we measured the extravasation of EB dye into the brain. Previous studies have shown that the increase in BBB permeability after brain injury has two temporal phases (Baskaya et al., 1997). Consistent with this, cortical impact injury shows an initial increase in permeability that can be detected at $6 \mathrm{~h}$ after injury and is followed by a gradual rise in permeability that peaks between 1 and $3 \mathrm{~d}$ after injury (Fig. $4 A$, Injury-Veh; $n=6$ per time point). The secondary phase of EB extravasation was significantly reduced in animals receiving a $6 \mathrm{~h}$ postinjury injection of $5 \mathrm{mg} / \mathrm{kg}$ sulforaphane (Fig. $4 A$, Injury-SUL; $n=5$ per time point). To examine whether the early rise in BBB permeability is solely attributable to mechanical damage, preinjury administration (18 h before injury) of the drug was performed. It was found that EB extravasation was significantly reduced at $6 \mathrm{~h}$ after injury (Injury-Veh, $100 \pm 19.54 \%, n=5$; Injury-SUL, $52.54 \pm 6.22 \%, n=6$; data not shown; $p<0.05$ ). Because EB dye is reflective of albumin extravasation, we examined whether the permeability of another dye of smaller molecular weight is also reduced in response to sulforaphane treatment. Figure $4 B$ shows that, similar to EB, the extravasation of fluorescein (376.28 molecular weight) into the brain measured $24 \mathrm{~h}$ after injury is significantly reduced in injured animals treated with sulforaphane compared with that detected in vehicle-treated injured animals ( $n=5$ per condition). The reduction in EB extravasation was not observed in animals receiving $1 \mathrm{mg} / \mathrm{kg}$ sulforaphane (Fig. $4 C$ ), a dose that was ineffective in inducing Nrf2-driven gene expression, and was also below the effective dose for increasing NQO1 enzyme activity (Fig. $1 C, D)$.

We have shown previously (Fig. $1 F$ ) that the induction of cytoprotective gene mRNAs by sulforaphane treatment can be blocked by intracerebroventricular infusion of ARE-containing decoy oligonucleotides. We next tested whether ARE oligonucleotides will influence the protection offered by sulforaphane administration. As shown in Figure 5, the protection of occludin and vWF immunoreactivity (Fig. $5 A ; n=4$ per condition) and 
A
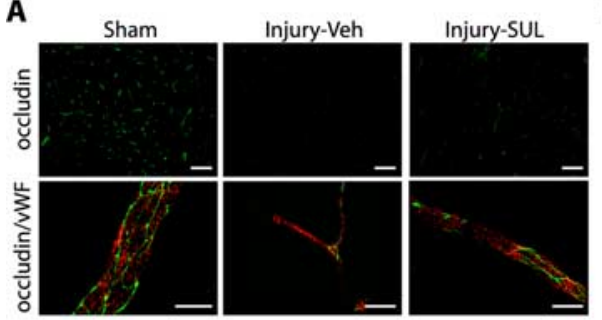

B

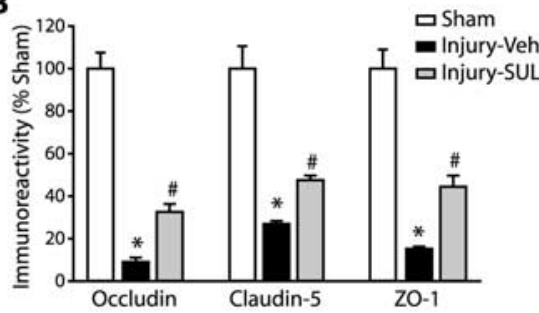

C Sham
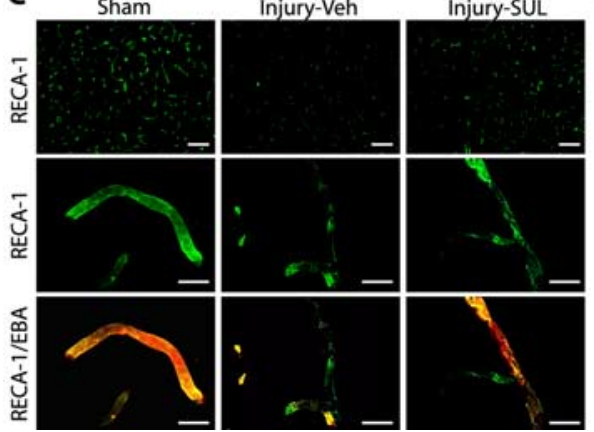

D

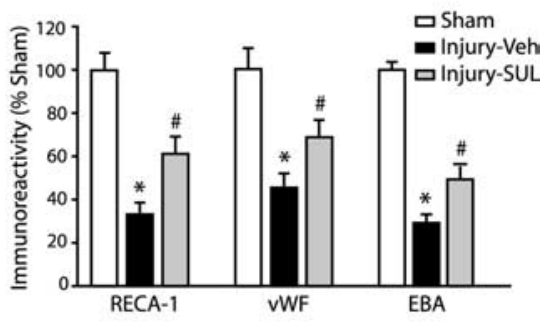

Figure 3. Sulforaphane attenuates the loss of tight junction proteins and endothelial cell markers after brain injury. $\boldsymbol{A}$, Representative low-magnification confocal images demonstrating occludin immunoreactivity (top row) in sham and $24 \mathrm{~h}$ injured animals. High-magnification confocal images of occludin-positive endothelial cells, as identified by vWF immunoreactivity are shown in the bottom row. $\boldsymbol{B}$, Summary data showing that the brain injury-associated losses of the tight junction proteins occludin and claudin-5 and the anchoring protein 20-1 are significantly attenuated by sulforaphane. $\boldsymbol{C}$, Representative confocal images demonstrating RECA-1 (top and middle rows) immunoreactivity. Double-label immunohistochemistry demonstrating RECA-1 and EBA immunoreactivities are shown in the bottom row. D, Summary data showing that the loss of RECA-1 and vWF and the barrier integrity marker EBA are attenuated after administration of sulforaphane. Data are presented as the mean \pm SEM. ${ }^{*} p<$ 0.05 compared with sham group. ${ }^{\#} p<0.05$ compared with vehicle group. Scale bars: top row in $\boldsymbol{A}$ and $\boldsymbol{C}, 100 \mu \mathrm{m}$; bottom row in $\boldsymbol{A}$ and middle and bottom row in $\boldsymbol{C}, 20 \mu \mathrm{m}$. Veh, Vehicle; SUL, sulforaphane.

BBB permeability (Fig. $5 B ; n=6$ per condition) observed after sulforaphane treatment was blocked in animals that received preinjury ARE oligonucleotide. Because accumulation of circulating fluid as a result of compromised BBB integrity can contribute to cerebral edema after brain injury, we assessed whether sulforaphane treatment could reduce brain edema and whether this effect was dependent on Nrf2. Figure $5 C(n=6$ per condition) shows that administration of sulforaphane $15 \mathrm{~min}$ after injury reduced cerebral edema measured $24 \mathrm{~h}$. This reduction was mediated by Nrf2 because rats infused with the ARE, but not AREmut, oligonucleotides did not benefit from sulforaphane administration. It has been demonstrated previously that the gene induction capacity of sulforaphane is blunted in $n r f 2^{-1-}$ mice (McMahon et al., 2001). Figure 5D (left) shows that the protective effect of sulforaphane in reducing BBB permeability after brain injury was blocked in $n r f 2$ null mice. Consistent with our results obtained using the ARE-containing oligonucleotides, knock-out of nrf2 not only blocked the protective effect of sulforaphane but was associated with increased EB extravasation compared with wild-type controls ( $n=3$ per condition). This suggests that mice lacking Nrf2 may have an increased susceptibility to injury compared with wild-type mice. To examine this possibility, groups of wild-type and $n r f 2^{-1-}$ mice were injured, and $E B$ extravasation was examined. Figure $5 D$ (right) shows that EB extravasation in $n r f 2$ knock-out animals $(n=4)$ was elevated compared with wild-type controls $(n=3)$. This increase was consistent in magnitude to that observed in sulforaphane-treated $n r f 2^{-/-}$mice, indicating that, in the absence of Nrf2, sulforaphane has no benefit on postinjury barrier integrity.

\section{Discussion}

Proper functioning of the blood-brain barrier is critical for maintaining brain homeostasis and function. Its dysfunction as a result of traumatic brain injury leads to the development of secondary pathological processes that can profoundly influence outcome. The present investigation into brain injury-induced $\mathrm{BBB}$ compromise revealed two key findings: (1) brain injury causes loss of endothelial cells and tight junction proteins that contribute to enhanced BBB permeability, and (2) the enhanced expression of endogenous Nrf2driven genes markedly attenuates brain injury-induced BBB disruption.

Previous studies have shown that processes such as oxidative damage, inflammation, and activation of matrix metalloproteases play key roles in the disruption of the BBB after injury (Chan et al., 1984; Povlishock and Kontos, 1992; Mikawa et al., 1996; Petty and Lo, 2002; Danton and Dietrich, 2003). Consistent with previous reports, we observed that traumatic brain injury results in a biphasic opening of the BBB: an early phase occurring within hours of the insult, and a secondary phase that peaks $\sim 1-3 \mathrm{~d}$ after injury. It is thought that the initial opening of the barrier results from mechanical damage to brain capillaries, with the late phase being primarily attributable to secondary processes. Interestingly, preinjury administration of sulforaphane significantly reduced Evans Blue extravasation during the acute phase, suggesting that mechanical damage is not the sole cause of this acute phase of barrier compromise. Consistent with this, measurement of free radical production has shown that there is an immediate increase in hydroxyl radicals after brain injury that is followed by an increase in lipid peroxidation and opening of the BBB (Smith et al., 1994). When the administration of sulforaphane was delayed to $6 \mathrm{~h}$ after injury, it markedly reduced the secondary phase of BBB permeability. This reduction in BBB permeability was associated with a protection of two key components of the BBB: endothelial cells and tight junction proteins. Our microvessel preparations demonstrate that the induction of mRNAs observed after sulforaphane administration can occur in vascular endothelial cells, suggesting that the effect on the BBB in vivo is a primary effect of sulforaphane. However, it is likely that the systemic administration paradigm used herein results in increased enzyme levels in many different cell types. Because the function of the neurovascular unit is dependent on neuronal, glial, and endothelial cell health and function, it is difficult to separate the influence of one of these cell types from effects of the other. In addition, it is unclear whether the protection offered by sulforaphane on tight junction protein loss occurred independent from, or as a result of, reduced endothelial cell loss. Our immunohistochemistry results showed that, after injury, von Willebrand factor-positive vessels could be detected that were occludin negative, suggesting that a temporal difference between endothelial cell death and tight junction protein loss may exist. However, we cannot rule out that occludin 
A
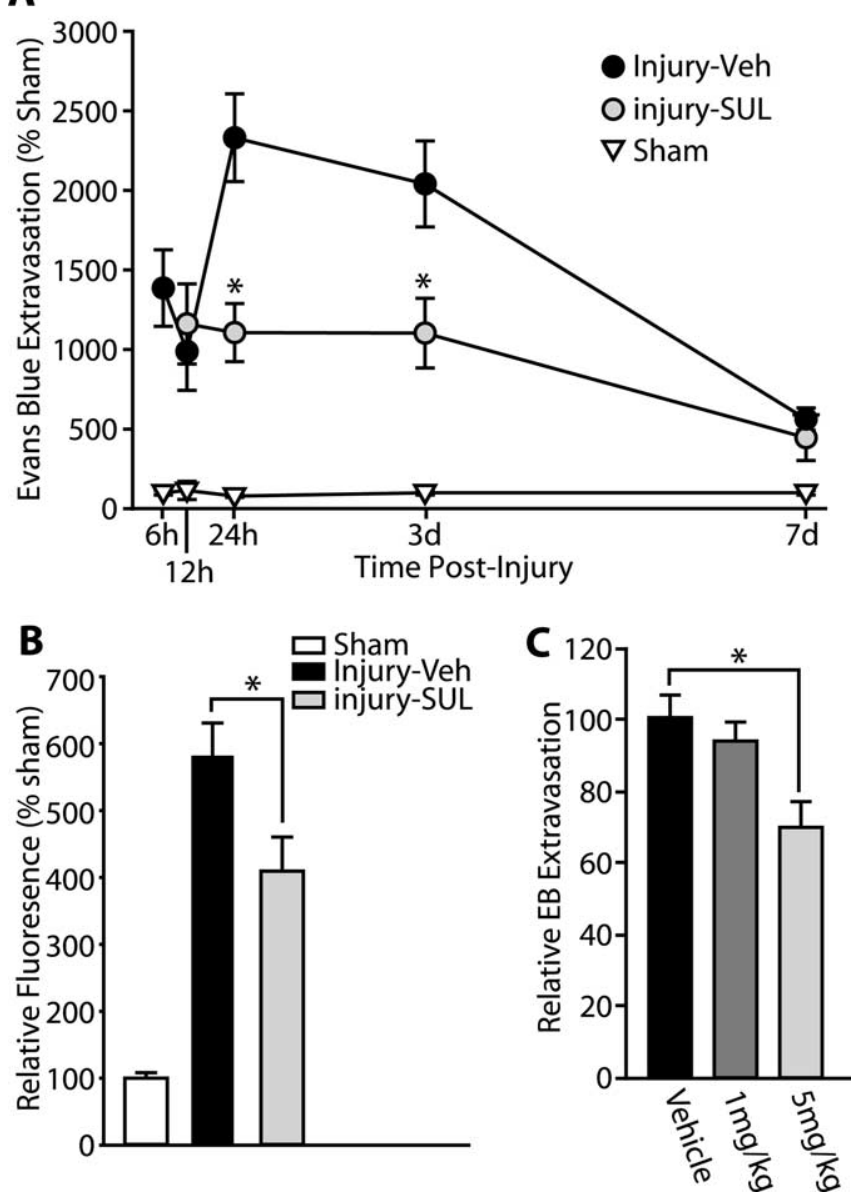

Figure 4. Postinjury sulforaphane administration decreases blood-brain barrier permeability after TBI. $A$, Time course of EB dye extravasation after TBI in sham and injured animals. Administration of sulforaphane $6 \mathrm{~h}$ after injury significantly decreases EB extravasation measured at both $24 \mathrm{~h}$ and $3 \mathrm{~d}$. B. Six hours postinjury administration of sulforaphane significantly decreases the BBB permeability to a low-molecular-weight compound (fluorescein) at $24 \mathrm{~h}$ after injury. C, Sulforaphane at $5 \mathrm{mg} / \mathrm{kg}$, but not $1 \mathrm{mg} / \mathrm{kg}$, is able to attenuate EB extravasation at $24 \mathrm{~h}$ after injury. ${ }^{*} p<0.05$ compared with injury-vehicle group. Veh, Vehicle; SUL, sulforaphane.

degradation is simply an early event occurring in dying endothelial cells.

A number of studies have shown that the ability of sulforaphane to induce cytoprotective genes is mediated through the transcription factor Nrf2. In normal or unstimulated conditions, most of the Nrf2 protein is present in the cytoplasm of the cell (Kang et al., 2004). It is held there by an adaptor protein called Keap1 (Itoh et al., 1999) that not only sequesters Nrf2 in the cytoplasm but also targets it for proteosome-mediated degradation (Zhang and Hannink, 2003; Zhang et al., 2004). It has been reported that sulforaphane increases nuclear Nrf2 levels, possibly by suppressing Keap1-mediated proteosomal degradation (McMahon et al., 2003). Alternative models for Nrf2 activation by sulforaphane, such as protein kinase-mediated phosphorylation, have been proposed (Shen et al., 2005; Giudice and Montella, 2006). In addition, we cannot rule out the possibility that sulforaphane may act through an as yet undiscovered pathway to influence Nrf2 and protect neurovascular function.

One weakness associated with the present study is that we have not directly examined the levels or nuclear localization of Nrf2 protein after sulforaphane administration. However, sulfora- phane increased the mRNA levels of GST $\alpha 3$, GPx, and HO-1, three Nrf2-driven genes. These increases, as well as the protective effects of sulforaphane on endothelial cell death and BBB compromise, were blocked by ARE-containing decoy oligonucleotides but not by oligonucleotides carrying mutations of the ARE sequence. In addition to sequestering Nrf2, these oligonucleotides may also bind the related transcription factor Nrf1 (Itoh et al., 1999; Zipper and Mulcahy, 2002), making it difficult to specifically address a role for Nrf2. However, the protective effect of sulforaphane on BBB compromise was also blocked in mice lacking the $n r f 2$ gene, further implicating this transcription factor. Together, our results are consistent with an involvement of Nrf2mediated cytoprotective gene expression in preserving BBB integrity after injury by sulforaphane.

The measurement of BBB permeability in injured mice lacking the $n r f 2$ gene revealed an $\sim 25 \%$ increase in EB extravasation compared with injured wild-type controls. This increase in barrier permeability was also observed in our experiments using the ARE oligonucleotides. This suggests that the loss of Nrf2 increased the vulnerability of the BBB to injury. Previous studies using $n r f 2^{-/-}$mice have shown that $\mathrm{Nrf} 2$ regulates not only the inducible expression of numerous cytoprotective genes but also the basal expression of many of these genes (McMahon et al., 2001). Therefore, the loss of Nrf2 may increase the susceptibility of the BBB to injury by either reducing the basal levels of cytoprotective proteins or by reducing the endogenous response of the endothelial cells to the injury.

Disruption of the BBB after insults to the CNS such as trauma and stroke or neurodegenerative conditions such as Alzheimer's disease is beginning to be an area of intensive research (Petty and Lo, 2002; Zlokovic, 2002). This attention is attributable to the recent appreciation that neuronal function is intimately linked to vascular function, acting together to control brain function and pathology. Because there is no clinically proven method for limiting brain damage after trauma, the potential clinical utility of Nrf2-activating compounds is encouraging. One advantage of activating Nrf2 as a potential treatment strategy is that a brief treatment can increase the expression of multiple protective proteins that can last for hours to days. For instance, increases in several glutathione-related enzymes have been reported to last over $120 \mathrm{~h}$ after exposure of cells to sulforaphane (Gao et al., 2001). This suggests that sulforaphane and/or related compounds, by inducing cell-protective genes in multiple cells types, including endothelial cells, astrocytes, and neurons, may effectively protect against several of the pathophysiological consequences of brain injury.

\section{References}

Alford MF, Masliah E, Hansen LA, Terry RD (1994) A simple dotimmunobinding assay for quantification of synaptophysin-like immunoreactivity in human brain. J Histochem Cytochem 42:283-287.

Baskaya MK, Rao AM, Dogan A, Donaldson D, Dempsey RJ (1997) The biphasic opening of the blood-brain barrier in the cortex and hippocampus after traumatic brain injury in rats. Neurosci Lett 226:33-36.

Chan PH, Schmidley JW, Fishman RA, Longar SM (1984) Brain injury, edema, and vascular permeability changes induced by oxygen-derived free radicals. Neurology 34:315-320.

Danton GH, Dietrich WD (2003) Inflammatory mechanisms after ischemia and stroke. J Neuropathol Exp Neurol 62:127-136.

Dash PK, Moore AN, Dixon CE (1995) Spatial memory deficits, increased phosphorylation of the transcription factor CREB, and induction of the AP-1 complex following experimental brain injury. J Neurosci 15:2030-2039.

Dash PK, Orsi SA, Moore AN (2005) Sequestration of serum response factor 
A

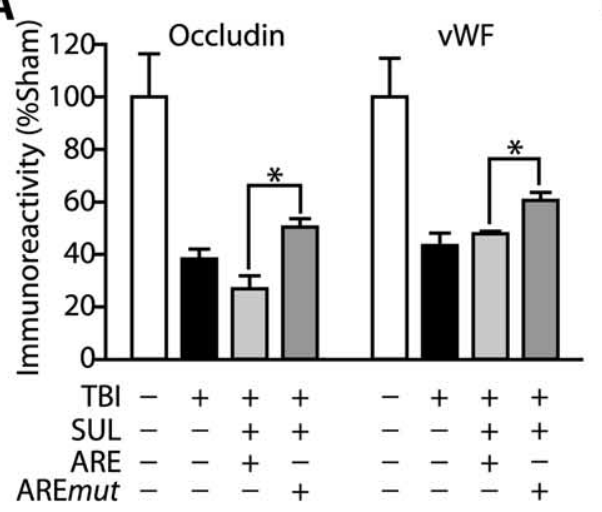

B

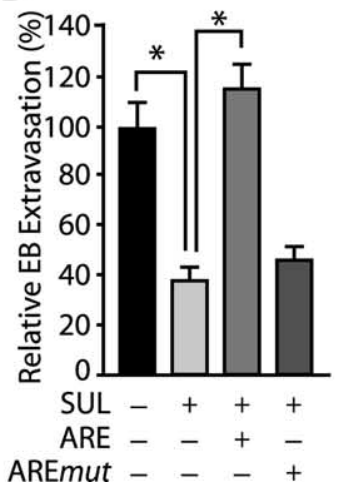

C

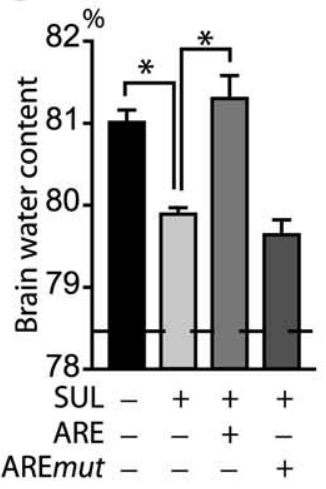

D

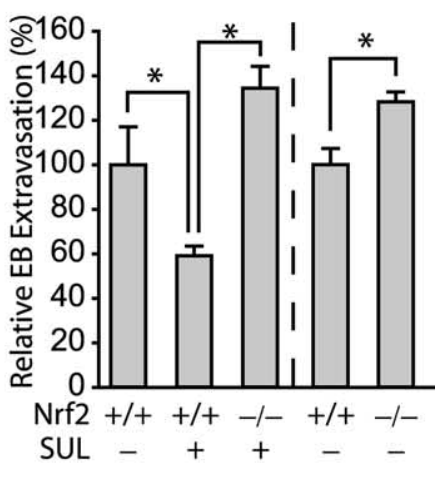

Figure 5. The protective effect of sulforaphane requires Nrf2. A, Summary data showing that intracerebroventricular infusion of the ARE decoy, but not the AREmut oligonucleotide, blocked the protective effect of sulforaphane (SUL) given 15 min after injury on occludin and vWF immunoreactivities in injured animals. $\boldsymbol{B}$, The protective effect of sulforaphane on EB extravasation in injured animals is blocked by intracerebroventricular infusion of ARE but not AREmut oligonucleotides. C, Postinjury administration of sulforaphane significantly reduces cerebral edema measured at $24 \mathrm{~h}$. Intracerebroventricular infusion of ARE, but not AREmut, oligonucleotides blocks the protective effect of sulforaphane administered 15 min after injury. $\boldsymbol{D}$, The protective effect of sulforaphane on EB extravasation is not present in mice lacking the nrf2 gene. In addition, the $n r f 2$ knock-out mice are more susceptible to injury-induced EB extravasation. Data are presented as the mean \pm SEM ${ }^{*} p<0.05$.

in the hippocampus impairs long-term spatial memory. J Neurochem 93:269-278.

DeWitt DS, Prough DS (2003) Traumatic cerebral vascular injury: the effects of concussive brain injury on the cerebral vasculature. J Neurotrauma 20:795-825.

Dixon CE, Clifton GL, Lighthall JW, Yaghmai AA, Hayes RL (1991) A controlled cortical impact model of traumatic brain injury in the rat. J Neurosci Methods 39:253-262.

Fishman RA (1975) Brain edema. N Engl J Med 293:706-711.

Gao X, Dinkova-Kostova AT, Talalay P (2001) Powerful and prolonged protection of human retinal pigment epithelial cells, keratinocytes, and mouse leukemia cells against oxidative damage: the indirect antioxidant effects of sulforaphane. Proc Natl Acad Sci USA 98:15221-15226.

Giudice A, Montella M (2006) Activation of the Nrf2-ARE signaling pathway: a promising strategy in cancer prevention. BioEssays 28:169-181.

Hu R, Hebbar V, Kim BR, Chen C, Winnik B, Buckley B, Soteropoulos P, Tolias P, Hart RP, Kong AN (2004) In vivo pharmacokinetics and regulation of gene expression profiles by isothiocyanate sulforaphane in the rat. J Pharmacol Exp Ther 310:263-271.

Hu R, Xu C, Shen G, Jain MR, Khor TO, Gopalkrishnan A, Lin W, Reddy B, Chan JY, Kong AN (2006) Gene expression profiles induced by cancer chemopreventive isothiocyanate sulforaphane in the liver of C57BL/6J mice and C57BL/6J/Nrf2 (-/-) mice. Cancer Lett 243:170-192.

Itoh K, Wakabayashi N, Katoh Y, Ishii T, Igarashi K, Engel JD, Yamamoto M (1999) Keap1 represses nuclear activation of antioxidant responsive elements by Nrf2 through binding to the amino-terminal Neh2 domain. Genes Dev 13:76-86.

Kang MI, Kobayashi A, Wakabayashi N, Kim SG, Yamamoto M (2004) Scaffolding of Keap1 to the actin cytoskeleton controls the function of Nrf2 as key regulator of cytoprotective phase 2 genes. Proc Natl Acad Sci USA 101:2046-2051.

Kobayashi M, Yamamoto M (2006) Nrf2-Keap1 regulation of cellular defense mechanisms against electrophiles and reactive oxygen species. Adv Enzyme Regul 46:113-140.

Lee JM, Calkins MJ, Chan K, Kan YW, Johnson JA (2003) Identification of the NF-E2-related factor-2-dependent genes conferring protection against oxidative stress in primary cortical astrocytes using oligonucleotide microarray analysis. J Biol Chem 278:12029-12038.

Lyeth BG, Liu S, Hamm RJ (1993) Combined scopolamine and morphine treatment of traumatic brain injury in the rat. Brain Res 617:69-75.

Masliah E, Ellisman M, Carragher B, Mallory M, Young S, Hansen L, DeTeresa R, Terry RD (1992) Three-dimensional analysis of the relationship between synaptic pathology and neuropil threads in Alzheimer disease. J Neuropathol Exp Neurol 51:404-414.

McIntosh TK, Soares H, Thomas M, Cloherty K (1990) Development of regional cerebral oedema after lateral fluid-percussion brain injury in the rat. Acta Neurochir Suppl (Wien) 51:263-264.
McMahon M, Itoh K, Yamamoto M, Chanas SA, Henderson CJ, McLellan LI, Wolf CR, Cavin C, Hayes JD (2001) The Cap'n'Collar basic leucine zipper transcription factor Nrf2 (NF-E2 p45-related factor 2) controls both constitutive and inducible expression of intestinal detoxification and glutathione biosynthetic enzymes. Cancer Res 61:3299-3307.

McMahon M, Itoh K, Yamamoto M, Hayes JD (2003) Keap1-dependent proteasomal degradation of transcription factor $\mathrm{Nrf} 2$ contributes to the negative regulation of antioxidant response element-driven gene expression. J Biol Chem 278:21592-21600.

Meaney DF, Ross DT, Winkelstein BA, Brasko J, Goldstein D, Bilston LB, Thibault LE, Gennarelli TA (1994) Modification of the cortical impact model to produce axonal injury in the rat cerebral cortex. J Neurotrauma 11:599-612.

Mikawa S, Kinouchi H, Kamii H, Gobbel GT, Chen SF, Carlson E, Epstein CJ, Chan PH (1996) Attenuation of acute and chronic damage following traumatic brain injury in copper, zinc-superoxide dismutase transgenic mice. J Neurosurg 85:885-891.

Oby E, Janigro D (2006) The blood-brain barrier and epilepsy. Epilepsia 47:1761-1774.

Petty MA, Lo EH (2002) Junctional complexes of the blood-brain barrier: permeability changes in neuroinflammation. Prog Neurobiol 68:311-323.

Pi J, Qu W, Reece JM, Kumagai Y, Waalkes MP (2003) Transcription factor Nrf2 activation by inorganic arsenic in cultured keratinocytes: involvement of hydrogen peroxide. Exp Cell Res 290:234-245.

Povlishock JT, Kontos HA (1992) The role of oxygen radicals in the pathobiology of traumatic brain injury. Hum Cell 5:345-353.

Qiu J, Hu X, Nesic O, Grafe MR, Rassin DK, Wood TG, Perez-Polo JR (2004) Effects of NF-kappaB oligonucleotide "decoys" on gene expression in P7 rat hippocampus after hypoxia/ischemia. J Neurosci Res 77:108-118.

Redell JB, Moore AN, Dash PK (2003) Expression of the prodynorphin gene after experimental brain injury and its role in behavioral dysfunction. Exp Biol Med (Maywood) 228:261-269.

Rosenstein JM, Krum JM, Sternberger LA, Pulley MT, Sternberger NH (1992) Immunocytochemical expression of the endothelial barrier antigen (EBA) during brain angiogenesis. Brain Res Dev Brain Res 66:47-54.

Shapira Y, Setton D, Artru AA, Shohami E (1993) Blood-brain barrier permeability, cerebral edema, and neurologic function after closed head injury in rats. Anesth Analg 77:141-148.

Shen G, Jeong WS, Hu R, Kong AN (2005) Regulation of Nrf2, NF-kappaB, and AP- 1 signaling pathways by chemopreventive agents. Antioxid Redox Signal 7:1648-1663.

Shohami E, Novikov M, Mechoulam R (1993) A nonpsychotropic cannabinoid, HU-211, has cerebroprotective effects after closed head injury in the rat. J Neurotrauma 10:109-119.

Shohami E, Novikov M, Horowitz M (1994) Long term exposure to heat 
reduces edema formation after closed head injury in the rat. Acta Neurochir Suppl (Wien) 60:443-445.

Smith SL, Andrus PK, Zhang JR, Hall ED (1994) Direct measurement of hydroxyl radicals, lipid peroxidation, and blood-brain barrier disruption following unilateral cortical impact head injury in the rat. J Neurotrauma 11:393-404.

Thimmulappa RK, Mai KH, Srisuma S, Kensler TW, Yamamoto M, Biswal S (2002) Identification of Nrf2-regulated genes induced by the chemopreventive agent sulforaphane by oligonucleotide microarray. Cancer Res 62:5196-5203.

Verschure PJ, van Marle J, Joosten LA, Van den Berg WB (1994) Localization and quantification of the insulin-like growth factor- 1 receptor in mouse articular cartilage by confocal laser scanning microscopy. J Histochem Cytochem 42:765-773.

Wagnerova J, Cervenakova L, Balabanov R, Zitron I, Dore-Duffy P (2002) Cy- tokine regulation of E-selectin in rat CNS microvascular endothelial cells: differential response of CNS and non-CNS vessels. J Neurol Sci 195:51-62.

Zhang DD, Hannink M (2003) Distinct cysteine residues in Keap1 are required for Keap1-dependent ubiquitination of Nrf2 and for stabilization of Nrf2 by chemopreventive agents and oxidative stress. Mol Cell Biol 23:8137-8151.

Zhang DD, Lo SC, Cross JV, Templeton DJ, Hannink M (2004) Keap1 is a redox-regulated substrate adaptor protein for a Cul3-dependent ubiquitin ligase complex. Mol Cell Biol 24:10941-10953.

Zipper LM, Mulcahy RT (2002) The Keap1 BTB/POZ dimerization function is required to sequester Nrf2 in cytoplasm. J Biol Chem 277:36544-36552.

Zlokovic BV (2002) Vascular disorder in Alzheimer's disease: role in pathogenesis of dementia and therapeutic targets. Adv Drug Deliv Rev 54:15531559. 